\title{
ANÁLISIS DE MÉTODOS DE DELIMITACIÓN DE INFRAESTRUCTURA VERDE EN UN TERRITORIO ALTAMENTE FRAGMENTADO
}

\author{
$\underline{\text { Rocío Losada }}^{a^{*}}$, Andrés M. García ${ }^{b}$ Inés Santé-Riveira $^{b}$ y David Miranda ${ }^{b}$ \\ ${ }^{a}$ Departamento de Enxeñería Agroforestal. Universidad de Santiago de Compostela, Lugo, \\ rocio.losada@rai.usc.es. ${ }^{b}$ Departamento de Enxeñería Agroforestal. Universidad de Santiago de \\ Compostela, Lugo.
}

\begin{abstract}
Resumen
La Estrategia de Biodiversidad 2030 de la Unión Europea prevé la creación de infraestructuras verdes para mitigar los impactos del cambio climático en la biodiversidad. En este sentido, la Comisión Europea define las infraestructuras verdes como una red de espacios naturales destinada a fomentar la provisión de múltiples servicios ecosistémicos que son fundamentales para preservar los valores naturales y el desarrollo de actividades socioeconómicas clave para el bienestar de la población. Los estados miembros, deben por lo tanto incorporar estas infraestructuras a su planeamiento territorial. No obstante, hay pocas metodologías disponibles para la delimitación de infraestructura verde, y la mayoría se basan en métodos de análisis multicriterio que tienen en cuenta el potencial del territorio de proveer servicios ecosistémicos. Estos potenciales de provisión se suelen estimar a partir de las coberturas del suelo debido a la escasez de información específica. En regiones donde las coberturas están muy fragmentadas, el uso de estos métodos produce elementos de infraestructura verde con límites irregulares y fragmentados, donde es más difícil gestionar el territorio para garantizar un desarrollo socioeconómico en armonía con la preservación de los valores naturales. Por otro lado, la estructura del paisaje, y no solo las coberturas del suelo, puede tener una gran influencia en el potencial de provisión de varios servicios ecosistémicos. En el presente trabajo se propone un método para delimitar elementos de infraestructura verde basándose en el potencial de provisión de servicios ecosistémicos de unidades del paisaje. Este método se comparará con un método basado en análisis multicriterio para analizar si se obtienen elementos de infraestructura verde menos fragmentados y con límites más regulares que sigan garantizando un potencial de provisión alto para varios servicios ecosistémicos.
\end{abstract}

Palabras clave: delimitación de infraestructura verde, unidades de paisaje, cambio climático

\section{Introducción}

En la Estrategia Biodiversidad 2030 de la Unión Europea (COM2020) se identifica la Infraestructura Verde (IV) como una de las herramientas para abordar los impactos del cambio climático en los valores naturales. En esta línea la Comisión Europea define la IV como una red de espacios naturales planificada para ofrecer una amplia gama de servicios ecosistémicos clave tanto para el desarrollo de actividades socioeconómicas que garantizan el bienestar humano como para preservar los valores naturales (COM2013). En línea con esto, los estados miembros de la Unión Europea deben desarrollar sus propias IV e integrarlas en su planificación.

España ya ha hecho su estrategia IV (Ministerio para la Transición Ecológica 2020). En las bases científico técnicas de la misma (Valladares et al. 2017) se especifica qué elementos deben de formar la IV: Áreas núcleo centradas en la conservación de los valores naturales, corredores ecológicos que conecten las áreas núcleo, áreas de amortiguación para mitigar impactos en los valores naturales de áreas núcleo y corredores ecológicos, elementos multifuncionales para la producción sostenible de recursos naturales y elementos urbanos. Para poder delimitar elementos de IV que garantice la provisión de múltiples Servicios Ecosistémicos (SE), es fundamental considerar el potencial de provisión de estos SE en el territorio (Ruckelshaus et al. 2015).

Sin embargo, las metodologías para delimitar elementos de IV multifuncionales que faciliten su inclusión en los planes territoriales son todavía escasas (Rodríguez Espinosa y Aguilera Benavente 2016). Siendo los métodos que se basan en el Análisis de Evaluación Multicriterio los más ampliamente utilizados (Kopperoinen, et al. 2014, Liquete et al. 2015, Rodríguez Espinosa y Aguilera Benavente 2016). Una de las limitaciones a la hora del mapeo del potencial de provisión de una amplia gama de SE es la escasez de datos debido a su coste de adquisición. Esta limitación se suele superar utilizando mapas de cobertura terrestre como proxy de los ecosistemas presentes en un área, para determinar su potencial de provisión (Maes et al. 2016). No obstante, al utilizar mapas de potencial de provisión generados a partir de coberturas del suelo en métodos basados en evaluación multicriterio para delimitar IV en territorios con paisajes fragmentados se obtienen elementos de IV con límites irregulares y fragmentados. Es por lo tanto difícil hacer una gestión territorial en estas zonas que garantice el desarrollo de actividades socioeconómicas en equilibrio con la conservación de los valores naturales. 
Si bien, algunos SE están muy influenciados por la cobertura terrestre (Mitchell et al. 2015, 2014), existe una evidencia creciente de que la estructura del paisaje tiene efectos relevantes en la provisión de SE (Bodin et al. 2006, Syrbe y Walz 2012). Así, autores como (Qiu y Turner 2013) sugieren considerar áreas de paisaje más amplias en el momento de identificar áreas con alto potencial de proporcionar varios SE para evitar patrones fragmentados. Esto también permitiría el desarrollo de modelos de gestión que integren mejor las actividades socioeconómicas con la conservación de la biodiversidad.

\section{Objetivos}

El objetivo del presente trabajo es, testar un método basado en unidades del paisaje para delimitar zonas de amortiguación multifuncionales de límites más regulares y menos fragmentadas que faciliten la gestión del territorio, garantizando un desarrollo socioeconómico en consonancia con la preservación de los valores naturales en un territorio fragmentado.

\section{Metodología}

Se delimitaron elementos de IV destinados a mitigar impactos del cambio climático en zonas con alto valor natural de la IV en la comarca de Lugo (Galicia, España), como son las zonas de amortiguación multifuncionales. Para ello se emplearon dos metodologías: una basada en análisis de evaluación multicriterio (García et al. 2020) y la metodología propuesta en este trabajo que considera el potencial de provisión de SEs de las unidades del paisaje. Los SE considerados en las delimitaciones, son aquellos relacionados con la mitigación de impactos del cambio climático. Luego se analizó con cuál de las metodologías se producen elementos más regulares. Los análisis se realizaron considerando el área media y la densidad de borde de las zonas delimitadas, así como el potencial medio de provisión de SE dentro de las mismas.

\section{1. Área de estudio}

La comarca de Lugo se ubica en el interior rural de Galicia (España). La comarca se compone del municipio urbano de Lugo con 100000 habitantes y siete municipios rurales circundantes que albergan una población de 20000 habitantes. Lugo, junto con Orense son las capitales administrativas del interior rural gallego y concentran el sector servicios de estas zonas. El área de estudio, al igual que el resto de Galicia, presenta paisajes muy fragmentados debido a la alta dispersión poblacional y al minifundio. La comarca posee importantes valores naturales asociados principalmente a los ecosistemas fluviales y humedales de la cuenca del río Miño, en las orillas del cual se sitúa la ciudad de Lugo. La actividad económica principal en la zona es la agroganadera, aunque también existe un importante sector secundario asociado principalmente a la zona urbana. El cambio climático amenaza a las actividades económicas y a los valores naturales de la comarca. El posible incremento en la variación de la pluviometría y la temperatura pueden conllevar al arrastre de nutrientes de las zonas ganaderas y contaminantes de la zona urbana a los ecosistemas acuáticos, mayor impacto de plagas y enfermedades en los cultivos o mayor riesgo de incendios forestales que causen pérdidas económicas.

\subsection{Servicios ecosistémicos}

Los servicios ecosistémicos considerados para este estudio son aquellos que pueden contrarrestar los efectos del cambio climático en los valores naturales de la comarca y en las actividades socioeconómicas. Estos son, teniendo en cuenta la clasificación del CICES (Haines-Young y Potschin 2018): 2.1.1.2 Filtración, secuestro, almacenamiento o acumulación de contaminantes por microorganismos, algas, plantas y animales. 2.2.1.1 Control de la erosión, 2.2.1.3 Regulación del ciclo hidrológico, 2.2.1.5 Protección contra incendios, 2.2.2.1 Polinización, 2.2.2.3 Mantenimiento de poblaciones y hábitats reproductores (incluida la protección del "pool" genético). 2.2.3.1 Control de plagas, 2.2.6.1 Regulación de la composición climática de la atmósfera y los océanos: absorción de carbono, captura de carbono a corto plazo, captura de carbono a largo plazo.

Las metodologías para mapear el potencial de provisión de los servicios ecosistémicos considerados para este estudio están descritas en García et al. (2020).

\subsection{Metodología de delimitación de zonas de amortiguación multifuncionales basada en unidades del paisaje}

Se tomó como referencia las unidades del paisaje delimitadas por Corbelle-Rico y Sánchez (2017). Se analizó cuáles son las unidades del paisaje con mayor potencial de provisión de SEs, tomando como referencia los mapas de provisión de SEs. Para ello, se emplearon estadísticas zonales con las que se estimó el potencial de provisión medio y el número medio de los SEs producidos por cada unidad. Se consideró que una zona provee un SE si esta tiene un potencial de provisión dentro del primer quintil de los potenciales de provisión calculados para ese SE en el área de estudio. 
Una vez identificadas las unidades que proveen un mayor número medio de SEs y tienen un potencial medio de provisión alto, se delinearon las zonas de amortiguación multifuncionales seleccionando las unidades con potenciales altos anexas a las zonas núcleo y corredores de la IV de la comarca.

Los resultados obtenidos con esta metodología se compararon con los resultados de aplicar la metodología de García et al. (2020); analizando la regularidad de las zonas delimitadas y el potencial de provisión de SEs de las mismas. Para ello se calcularon las métricas espaciales de densidad de borde y área media de las zonas delimitadas. También se determinó el potencial medio de provisión de SEs y el número medio de SEs producidos en las zonas delimitadas mediante estadísticas zonales.

\section{Resultados}

En el Mapa 1 se encuentran las zonas de amortiguación obtenidas con ambos métodos.

\section{Mapa 1. Zonas de amortiguación multifuncionales con los métodos de evaluación multicriterio (a) y} unidades del paisaje (b)

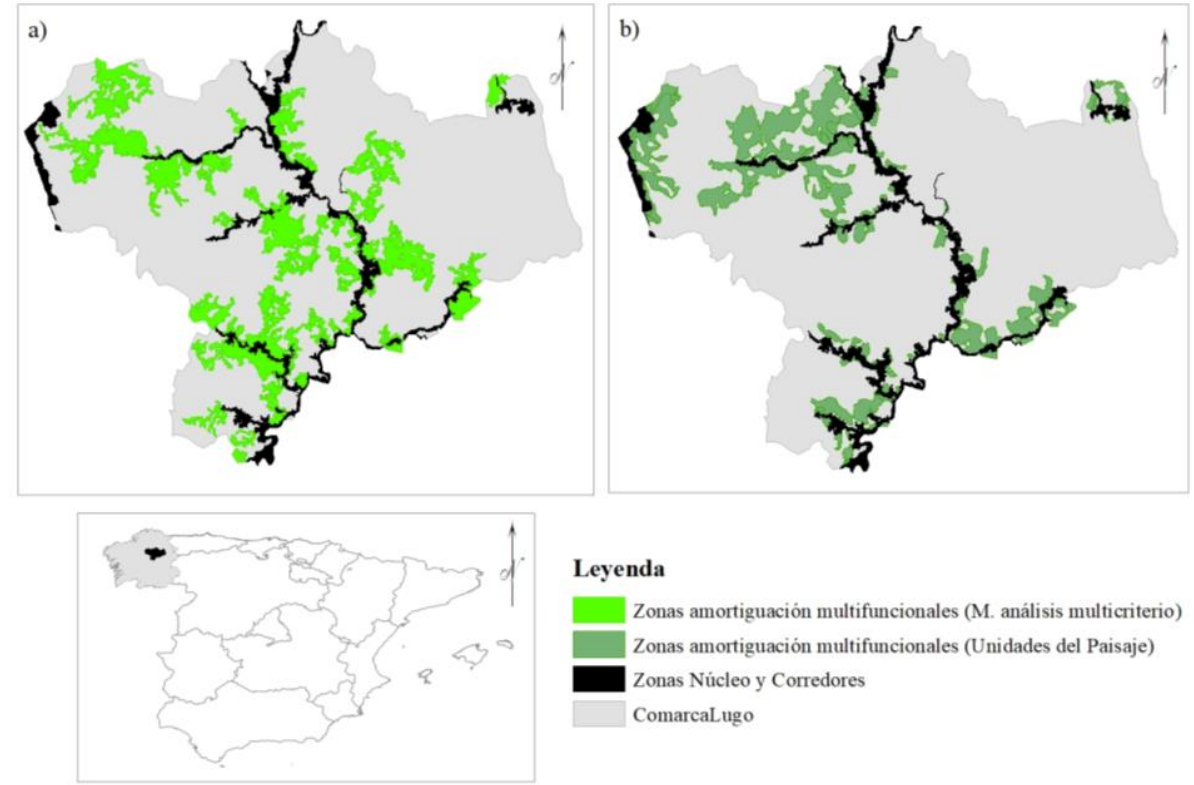

Algunas unidades del paisaje proveen un elevado número medio de SEs pero un bajo potencial medio de provisión. Este es el caso de la unidad de paisaje de rururbano diseminado. La diferencia puede deberse a las sinergias y antagonismos entre los distintos SEs.

En este sentido, para evitar conflictos al intentar maximizar la producción de varios SE antagónicos, se analizó la correlación entre los potenciales de provisión de los SE a través del índice de Spearman (Spearman, 1094) En base a esto, se ha visto que los SE de almacenamiento de carbono a medio plazo, control de la erosión, filtrado y ciclo hídrico presentan antagonismos con el SE de protección contra incendios. Por lo que se realizaron dos grupos de SE teniendo en cuenta la correlación entre los mismos: El primero compuesto por los SE de incendios, polinización, biodiversidad, control de plagas, captura de carbono y captura de carbono a largo plazo y el segundo, con el SE de almacenamiento a medio plazo, ciclo hídrico, control de la erosión y filtrado. El primer grupo está más relacionado con unidades de paisaje que tienen abundante biomasa mientras que el segundo con unidades del paisaje con menor biomasa como zonas agrícolas.

Finalmente, las unidades del paisaje seleccionadas fueron: lámina de agua, viñedo, bosque, agrosistema intensivo (plantación forestal) y agrosistema intensivo (mosaico agroforestal) por estar dentro de las cinco primeras unidades del paisaje que proveen un mayor número de SEs dentro de cada grupo de SEs. A mayores, estas unidades presentan un alto potencial medio de provisión de SEs en cada grupo. Por lo tanto, se delimitaron como zonas de amortiguación multifuncionales las unidades del paisaje anteriormente citadas que están anexas a zonas núcleo o corredores de la IV de la comarca.

Las zonas de amortiguación multifuncionales delimitadas con las dos metodologías presentan áreas similares: en el caso de la metodología basada en unidades del paisaje la superficie delimitada supone un $12,78 \%$ del área total y en el caso del método de evaluación multicriterio un 15,47\%. Aunque las superficies son similares, en ambas metodologías es difícil controlar el área delimitada.

En relación a la regularidad y tamaño de las zonas delimitadas, el área media de las manchas es de 406 para la evaluación multicriterio y la densidad de borde 12,9. En el caso de la metodología basada en unidades del paisaje, estos valores son 126,61 y 3,08 respectivamente. 
Por último, solo el potencial medio de provisión del SE de biodiversidad es ligeramente superior en las zonas delimitadas basándose en las unidades del paisaje. Para el resto de SE los valores medios son superiores con el método de evaluación multicriterio. En el caso del número medio de SEs producidos en las zonas delimitadas, solo los SE de biodiversidad, captura de carbono y control de plagas son superiores en el método basados en unidades del paisaje.

\section{Conclusiones}

A la vista de los resultados, al considerar unidades del paisaje en la delimitación, se obtienen zonas multifuncionales de amortiguación con límites más regulares y menos fragmentadas que mantienen el potencial medio de provisión de SEs alto. También, las zonas delimitadas se distribuyen más uniformemente a lo largo del borde de corredores y zonas núcleo, permitiendo una mayor provisión de SEs a las zonas que los demandan. La metodología ha ayudado a identificar cuáles son las unidades de paisaje que proveen SE antagónicos. De esta manera se garantiza que ambos tipos de unidades estén presentes y provean una mayor cantidad de SEs. La combinación de ambos tipos de unidades en un mosaico puede maximizar tanto la protección contra incendios como los SE antagónicos más relacionados con coberturas forestales. Por último, las unidades de paisaje que presentan mayor potencial de provisión de SE corresponden con usos agrícolas y forestales intensivos que pueden menguar la capacidad de provisión servicios de los ecosistemas. Para evitarlo, será necesario fomentar en estas zonas modelos de gestión sostenibles que maximicen la provisión de SEs y los beneficios de la actividad socioeconómica asociados a estos usos. Al delimitar zonas con límites más regulares y menos fragmentadas, se disponen de áreas más grandes donde crear agrupaciones de propietarios que faciliten la transición a modelos de gestión forestal basados en especies de crecimiento lento que son más rentables a largo plazo y generan menos impactos ambientales.

\section{Agradecimientos}

Esta investigación, financiada por la Fundación Biodiversidad del Ministerio de Transición Ecológica de España, se realizó bajo el marco del proyecto "INVERCLIMA - Infraestructura Verde para la adaptación de la ordenación territorial al cambio climático".

\section{Bibliografía}

Bodin, Ö., Tengö, M., Norman, A., Lundberg, J., and Elmqvist, T. (2006) 'The Value Of Small Size: Loss Of Forest Patches And Ecological Thresholds In Southern Madagascar'. Ecological Applications 16 (2), 440-451

Corbelle-Rico, E. and Sánchez, J.M.T. (2017) 'Mapping Landscape Units in Galicia (Spain): A First Step for Assessment and Management?' Change and Adaptation in Socio-Ecological Systems [online] 3 (1). available from <https://www.degruyter.com/document/doi/10.1515/cass-2017-0008/html> [29 April 2021]

European Commission Communication of the Commission to the European Parliament, the Council, the European Economic and Social Committee and the Committee of the Regions. (n.d.) $E U$ Biodiversity Strategy for 2030: Bringing Nature Back into Our Lives,. COM/2020/380 final

European Commission Communication of the Commission to the European Parliament, the Council, the European Economica and Social Committee and the Committee of the Regions (n.d.) Green Infrastructure (GI) - Enhancing Europe's Natural Capital. COM(2013) 249 final

García, A.M., Santé, I., Loureiro, X., and Miranda, D. (2020) 'Spatial Planning of Green Infrastructure for Mitigation and Adaptation to Climate Change at a Regional Scale'. Sustainability 12 (24), 10525

Haines-Young, R. and Potschin, M.B. (2018) 'Common International Classification of Ecosystem Services (CICES) V5.1 and Guidance on the Application of the Revised Structure'. European Environemnt Agency: Copenhaguen, Denmark,

Kopperoinen, L., Itkonen, P., and Niemelä, J. (2014) 'Using Expert Knowledge in Combining Green Infrastructure and Ecosystem Services in Land Use Planning: An Insight into a New Place-Based Methodology'. Landscape Ecology 29 (8), 1361-1375

Liquete, C., Kleeschulte, S., Dige, G., Maes, J., Grizzetti, B., Olah, B., and Zulian, G. (2015) 'Mapping Green Infrastructure Based on Ecosystem Services and Ecological Networks: A Pan-European Case Study'. Environmental Science \& Policy 54, 268-280

Maes, J., Crossman, N., and Burkhard, B. (2016) Mapping Ecosystem Services. In: Routledge Handbook of Ecosystem Services. Potschin P, Haines-Young R, Fish R, Turner RK. Routlegde. London, 188-204 
Ministerio para la Transición Ecológica (2020) Estrategia Estatal de Infraestructura Verde y de la Conectividad y la Restauración Ecológicas: Guía metodológica para la identificación de la Infraestructura Verde de España.

Mitchell, M.G.E., Bennett, E.M., and Gonzalez, A. (2014) 'Forest Fragments Modulate the Provision of Multiple Ecosystem Services’. Journal of Applied Ecology 51 (4), 909-918

Mitchell, M.G.E., Suarez-Castro, A.F., Martinez-Harms, M., Maron, M., McAlpine, C., Gaston, K.J., Johansen, K., and Rhodes, J.R. (2015) 'Reframing Landscape Fragmentation's Effects on Ecosystem Services'. Trends in Ecology \& Evolution 30 (4), 190-198

Qiu, J. and Turner, M.G. (2013) 'Spatial Interactions among Ecosystem Services in an Urbanizing Agricultural Watershed'. Proceedings of the National Academy of Sciences 110 (29), 12149_ 12154

Rodríguez Espinosa, V.M. yAguilera Benavente, F. (2016) ¿ ¿Infraestructuras Verdes En La Planificación Territorial Española? 48(189), 399-418

Ruckelshaus, M., McKenzie, E., Tallis, H., Guerry, A., Daily, G., Kareiva, P., Polasky, S., Ricketts, T., Bhagabati, N., Wood, S.A., and Bernhardt, J. (2015) 'Notes from the Field: Lessons Learned from Using Ecosystem Service Approaches to Inform Real-World Decisions'. Ecological Economics 115, 11-21

Spearman, C (1094) The Proof and Measurement of Association between Two Things. American Journal of Psycology. 15 72-101

Syrbe, R.-U. and Walz, U. (2012) 'Spatial Indicators for the Assessment of Ecosystem Services: Providing, Benefiting and Connecting Areas and Landscape Metrics'. Ecological Indicators 21, 80-88

Valladares, F., Gil, P., Forner, A., and (Coord.) (2017) Bases Científico-Técnicas Para La Estrategia Estatal de Infraestructura Verde y de La Conectividad y Restauración Ecológicas. Madrid: Ministerio de Agricultura y Pesca, Alimentación y Medio Ambiente 